\title{
High-Resolution Phase-Contrast Imaging at XFELs
}

A. Schropp ${ }^{1}$, R. Hoppe ${ }^{2}$, V. Meier ${ }^{2}$, J. Patommel ${ }^{2}$, F. Seiboth ${ }^{2}$, Hae Ja Lee ${ }^{3}$, B. Nagler ${ }^{3}$, E. C. Galtier $^{3}$, B. Arnold ${ }^{3}$, J.B. Hastings ${ }^{3}$, and C. G. Schroer ${ }^{1}$

(1) Deutsches Elektronen-Synchrotron DESY, Hamburg, Germany

(2) Technische Universität Dresden, Germany

(3) SLAC National Accelerator Laboratory, USA

Current x-ray sources of the fourth generation, also denoted by x-ray free-electron lasers (XFELs), produce intense and short $\mathrm{x}$-ray pulses opening up completely new scientific possibilities for the investigation of fast dynamical processes [1,2]. In this way snapshots of the state of matter can be recorded at specific moments in time. However, some experiments require as well high spatial resolution in order to visualize physical processes occurring on short length scales. While the temporal resolution is determined by the pulse length, the spatial resolution in $\mathrm{x}$-ray imaging is mainly limited by the properties of the x-ray optics and diffraction effects.

We built an x-ray microscope for the Matter in Extreme Conditions (MEC) endstation of the LCLS, which is especially adapted to the XFEL environment, and used the setup to carry out different experiments based on magnified x-ray phase-contrast imaging. The method allows one to image a sample with high spatial resolution and high sensitivity to small density changes within a sample. The xray microscope is based on a set of Beryllium compound refractive lenses (Be-CRLs) creating a secondary x-ray source closely in front of a sample. The sample is positioned in the divergent $\mathrm{x}$-ray beam and a CCD detector records a magnified image of theilluminated area at a larger distance further downstream [cf. Fig. 1a)]. In a pump-probe schemean optical laser hits the samplefrom the side, which initiates a shock wave propagating into the material, and the XFEL pulse probes the state of the material shortly after. In Figs. 1b)-1e) a series of measured phase-contrast images recorded on Kapton at diff tdelay times between the optical and XFEL pulse is shown. Larger features such as the position of the shock front and structures behind it can be observed directly in the image. However, due to diffraction effects it is necessary to evaluate the phase-contrast images numerically using phase retrieval methods in order to obtain high-resolution images of the sample. In addition, a quantitative analysis requires a precise knowledge of the illumination wave fi to disentangle features already present in the incoming $\mathrm{x}$-ray beam from the actual object. Beam characterization was carried out using scanning coherent $x$-ray microscopy (ptychography). The method yields the wave fi distribution of the nano-focused XFEL beam with high spatial resolution (cf. Fig. 2) [3, 4].

In this contribution we report on current activities to implement high-resolution x-ray imaging at the Linac Coherent Light Source (LCLS) [5]. We demonstrate these new capabilities at the example of imaging of shock waves in different materials, outline the analysis procedure and discuss limitations related to fluctuations inbeamintensityand position, bandwidth of the XFEL beam [6] and dispersive properties of the x-ray optics. 
a)

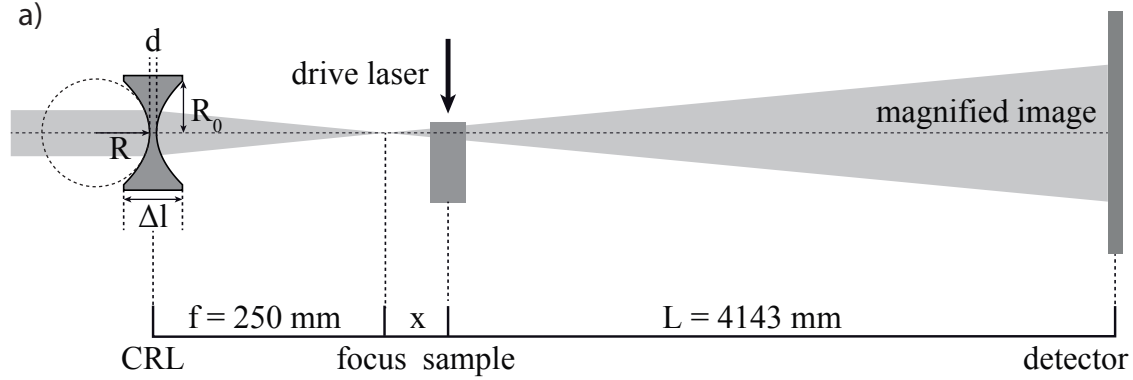

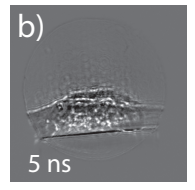

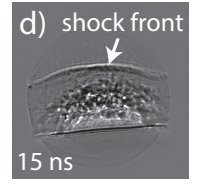

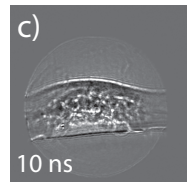

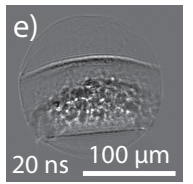

Figure 1: a) Experimental geometry for magnified phase-contrast imaging. b) - e) Phase contrast images measured on Kapton at different time delays between $5 \mathrm{~ns}$ and $20 \mathrm{~ns}$.

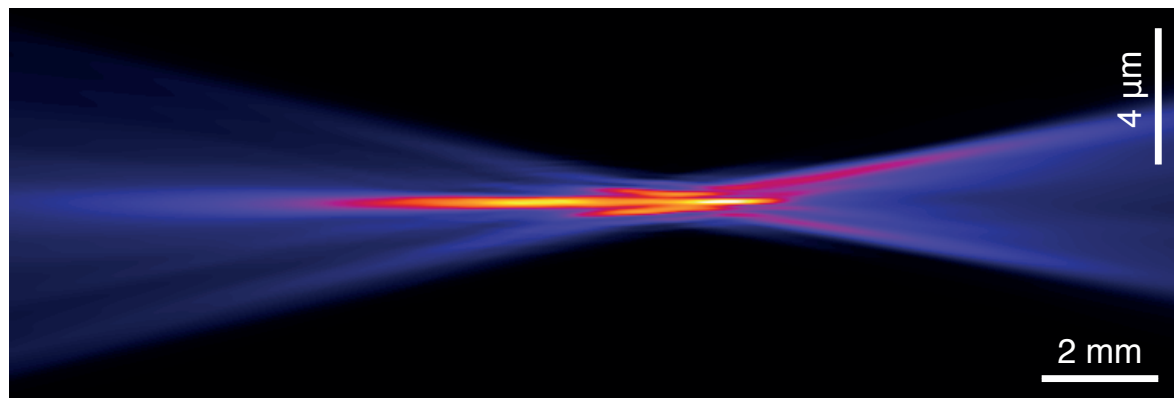

Figure 2: Vertical intensity profile of a $150 \mathrm{~nm}$-XFEL beam (FWHM).

\section{References}

[1] Chapman, H. N. et al. Femtosecond x-ray protein nanocrystallography. Nature 470, 73-U81 (2011).

[2] Berrah, N. et al. Double-core-hole spectroscopy for chemical analysis with an intense X-ray femtosecond laser. PNAS 108, 16912-16915 (2011).

[3] Schropp, A. et al. Full spatial characterization of a nanofocused x-ray free-electron laser beam by ptychographic imaging. Scientific Reports 3, 1633 (2013).

[4] Schropp, A. et al. Scanning coherent x-ray microscopy as a tool for xfel nanobeam characterization. Proc. SPIE 8849, 88490R (2013).

[5] Schropp, A. et al. Developing a platform for high-resolution phase contrast imaging of high pressure shock waves in matter. In Moeller, S. P., Yabashi, M. \& Hau-Riege, S. P. (eds.) Proc. of SPIE, vol. 8504, 85040F (SPIE, San Diego, 2012).

[6] Seiboth, F. et al. Focusing XFEL SASE pulses by rotationally parabolic refractive x-ray lenses. In J. Phys. Conf. Ser., vol. 499, 012004 (2014). 